\title{
Psychosocial Interventions to Improve Psychological Distress of Informal Caregivers of Cancer Patients: A Meta-analysis of Randomized Controlled Trial
}

\author{
Zheng Zhang, Shanshan Wang, Ziwei Liu, Zekai Li ${ }^{*}$ \\ School of Nursing, Jinan University, Guangzhou, China \\ Email address: \\ lizekaikl@163.com (Zekai Li) \\ ${ }^{*}$ Corresponding author

\section{To cite this article:} \\ Zheng Zhang, Shanshan Wang, Ziwei Liu, Zekai Li. Psychosocial Interventions to Improve Psychological Distress of Informal Caregivers of \\ Cancer Patients: A Meta-analysis of Randomized Controlled Trials. American Journal of Nursing Science. Vol. 9, No. 6, 2020 , pp. $459-465$. \\ doi: 10.11648/j.ajns.20200906.24
}

Received: December 6, 2020; Accepted: December 16, 2020; Published: December 22, 2020

\begin{abstract}
Objective: To evaluate the efficacy of psychosocial interventions designed to improve the psychological distress of informal caregivers of cancer patients compared with usual care. Methods: Nine relevant databases were searched from inception to November 2020. We included randomized controlled trials focused on comparing psychosocial interventions delivered to informal cancer caregivers with usual care. Study quality was evaluated with the Cochrane Risk of Bias Assessment Tool, and meta-analysis was performed using Review Manager statistical software. Results: Fifteen studies involving 1006 participants met the inclusion criteria, and several kinds of psychosocial interventions were applied to either cancer caregivers or patient-caregiver dyads. Compared to usual care, psychosocial interventions manifested positive, significant results in depression ( $\mathrm{SMD}=-0.47,95 \% \mathrm{CI}-0.71$ to $-0.24, \mathrm{P}<0.001$ ) and anxiety $(\mathrm{SMD}=-0.50,95 \% \mathrm{CI}-0.91$ to $-0.09, \mathrm{P}=0.02$ ). And the psychosocial interventions have no significant effect on general psychological distress ( $\mathrm{SMD}=-0.25,95 \% \mathrm{CI}-0.54$ to $0.05, \mathrm{P}=0.10)$ and subjective burden ( $\mathrm{SMD}=-0.06,95 \% \mathrm{CI}$ 0.26 to $0.14, \mathrm{P}=0.55$ ) of cancer caregivers. Conclusion: Psychosocial interventions designed for cancer caregivers can effectively reduce depression and anxiety, while no significant results were found in general distress and subjective burden of cancer caregivers. More studies with stronger methodological designs and larger samples are still needed in the future.
\end{abstract}

Keywords: Cancer, Caregivers, Psychosocial Intervention, Meta-analysis

\section{Introduction}

Worldwide, cancer is the second-leading cause of death, and about 1 in 6 deaths is due to cancer [1]. It is estimated that the number of death from cancer is 9.6 million deaths in 2018, according to the World Health Organization (WHO) [1]. As a result, there is an increasing number of informal caregivers who have to take the responsibility of taking care of cancer patients. Informal caregivers are families, close friends, or important people, providing care and support without economic benefits [2]. It is reported that informal caregivers experience a high level of psychological distress [3-4], which is detrimental to the well-being of both the caregivers and the people living with cancer. Thus, a growing body of literature has focused on a range of psychosocial interventions aiming at reducing the psychological distress of cancer caregivers [5-7].

There are various types of psychosocial intervention, including cognitive behavior therapy (CBT), psychoeducation, mindfulness, counseling, etc. Previously published meta-analysis studies reveal that psychosocial interventions have beneficial effects on improving the quality of life of both the cancer patients and their families [8], mood, and problem-solving skills [9]. So far, however, no consistent conclusion has been drawn about the effectiveness of psychosocial interventions to improve the psychological distress of cancer caregivers, though a large body of randomized controlled studies has been conducted. The 
purpose of the present study is to perform a meta-analysis of randomized controlled trials on the efficacy of psychosocial interventions aimed at improving the psychological distress of cancer caregivers.

\section{Methods}

\subsection{Search Methods}

Relevant electronic databases were searched to identify empirical studies published from inception to November 2020, including the Cochrane Library, PubMed, Embase, Web of Science, CINAHL, Scopus, CNKI, VIP, and Wan Fang database. The search strategy was to use medical subject headings and free terms together, including (psychosocial intervention OR cognitive behavior therapy OR psychotherapy OR psychoeducation OR mindfulness) AND (oncology OR cancer OR tumor OR carcinoma OR neoplasms) AND (caregiver* OR carer* OR family members OR relatives OR spouse). Additionally, reference lists of screened studies and pertinent reviews were examined to identify other relevant articles.

\subsection{Eligibility Criteria}

\subsubsection{Participants}

Participants were included if they were adults ( $\geq 18$ years) and they provided care and support for cancer patients without any economic benefits.

\subsubsection{Intervention}

Studies were included if informal caregivers of cancer patients in the trial group received psychosocial interventions, including CBT (Cognitive Behavior Therapy), psychoeducation therapy, psychotherapy, mindfulness, etc. We also included psychosocial interventions delivered to caregiver-patient dyads. The interventions were conducted at the hospital or participants' homes.

\subsubsection{Comparison}

Studies were included if the control group participants were provided with usual care.

\subsubsection{Outcomes}

The outcomes included general psychological distress, anxiety, depression, and subjective burden.

\subsubsection{Study Design}

Only randomized controlled trials were included.

\subsection{Data Extraction and Quality Assessment}

The data extraction was performed by the main author. Relevant information was recorded, including the study characteristics (year, author, country, study design), the intervention (type, sample size, duration, content), the comparison (type, sample size, duration, content), and outcome variables (general psychological distress, anxiety, depression, subjective burden). Two researchers independently conducted the quality assessments using the Cochrane Risk of Bias Assessment Tool, and the disagreements were resolved by consensus.

\subsection{Data Analysis}

Review Manager statistical software was used for the statistical analyses. Effect sizes between the trial and control groups were calculated, basing on means, standard deviations, standardized mean difference, and sample sizes. The random-effects model was used because it is a more conservative approach to explain different sources of variation among articles [10]. The homogeneity statistic Q was calculated to determine whether there is a lack of homogeneity among studies, and a significant $\mathrm{P}$ value means heterogeneity. The $\mathrm{I}^{2}$ statistic and its $95 \%$ CI were calculated to standardize the Q [11]. $\mathrm{I}^{2}$ ranges from $0 \%$ to $100 \%$, with large values $(>50 \%)$ indicating heterogeneity.

\section{Results}

\subsection{Study Characteristics}

Initial retrieval identified a total of 1717 papers, of which 474 were duplicates (Figure 1). After evaluating titles and abstracts, 146 papers were obtained and examined in full text. Finally, fifteen papers met the eligibility criteria and were included in the quantitative synthesis. The baseline characteristics of the included literature were documented in Table 1. In total, 1006 cancer caregivers were included in the meta-analysis. In these studies, different types of psychosocial interventions were used, including psychoeducation [7, 12-14], cognitive behavior therapy [1516], mindfulness therapy $[15,17]$, and other various kinds of psychosocial interventions $[5,15,18-20]$. Most of the interventions were carried out in the hospitals, and the intervention duration ranged widely from 3 weeks to 24 weeks.

Table 1. Characteristics of included studies.

\begin{tabular}{llll}
\hline Study & Sample Size (Trial/Control) & Setting & Intervention \\
\hline Baucom, 2009, USA & $14(8 / 6)$ & Hospital & RE (Relation Enhancement) \\
Boriji, 2017, Irian & $80(40 / 40)$ & Hospital & CBT (Cognitive Behavioral Therapy) \\
Couper, 2015, Australia & $62(30 / 32)$ & Hospital & CECT (Cognitive Existential Couple Therapy) \\
Hoesktra-weebers, 1998, Netherlands & $81(39 / 42)$ & Hospital & Psychoeducational Intervention \\
Kubo, 2019, USA & $31(17 / 14)$ & Home & M-health Mindfulness \\
Laundenslager, 2015, Australia & $101(48 / 90)$ & Hospital & PEPRR (Psychoeducation, Paced \\
Laundenslager, 2019, Australia & $155(75 / 80)$ & Respiration and Relaxation) \\
\hline
\end{tabular}




\begin{tabular}{llll}
\hline Study & Sample Size (Trial/Control) & Setting & Intervention \\
\hline & & & Respiration and Relaxation) \\
Manne, 2004, USA & $60(29 / 31)$ & $\begin{array}{l}\text { Cancer Center } \\
\text { Outpatient clinic; Inpatient } \\
\text { unit; Home }\end{array}$ & Stress Management Intervention \\
Marsland, 2012, USA & $37(23 / 14)$ & MBSR (Mindfulness-based Stress Reduction) \\
Melanie, 2017, Netherlands & $35(16,19)$ & Local University & ERT (Emotion Regulation Therapy) \\
O'Toole, 2020, Denmark & $80(43 / 37)$ & Hospital; Home & BPI (Brief Psychosocial Intervention) \\
Safarabadi-Farahani, 2016, Irian & $65(32 / 33)$ & Home & Family Connect Intervention \\
Shaw, 2016, Australia & $128(64 / 64)$ & Oncology center & Problem Solving Intervention \\
Toseland, 1995, USA & $78(40,38)$ & Hospital & CBT (Cognitive Behavioral Therapy) \\
Wu, 2018, China & $60(30 / 30)$ & & \\
\hline
\end{tabular}

Table 1. Continued.

\begin{tabular}{|c|c|c|c|}
\hline Study & Comparison & Duration & Outcome And Outcome Measure \\
\hline Baucom, 2009, USA & Usual Care & 12 weeks & Psychological Distress (BSI) \\
\hline Boriji, 2017, Irian & Usual Care & 12 weeks & Depression (DASS); Anxiety (DASS) \\
\hline Couper, 2015, Australia & Usual Care & 10 weeks & Psychological Distress (MHI) \\
\hline Hoesktra-weebers, 1998, Netherlands & Usual Care & 24 weeks & Psychological Distress (CHQ) \\
\hline Kubo, 2019, USA & Usual Care & 8 weeks & Psychological Distress (Distress Thermometer) \\
\hline Laundenslager, 2015, Australia & Usual Care & 3 weeks & $\begin{array}{l}\text { Depression (CES-D); Anxiety (STAI-State); Subjective burden (CRA); } \\
\text { Psychological Distress (CG-Distress) }\end{array}$ \\
\hline Laundenslager, 2019, Australia & Usual Care & 14 weeks & $\begin{array}{l}\text { Distress (CG-distress); Depression (CES-D); Anxiety (STAI-State); } \\
\text { Subjective Burden (CRA) }\end{array}$ \\
\hline Manne, 2004, USA & Usual Care & 6 weeks & Psychological Distress (MHI) \\
\hline Marsland, 2012, USA & Usual Care & $12 \sim 18$ weeks & Depression (BDI); Anxiety (STAI) \\
\hline Melanie, 2017, Netherlands & Usual Care & 8 weeks & Psychological distress (HADS) \\
\hline O’Toole, 2020, Denmark & Usual Care & 8 weeks & Psychological Distress (HADS) \\
\hline Safarabadi-Farahani, 2016, Irian & Usual Care & 5 weeks & Emotional Burden (CQOLC) \\
\hline Toseland, 1995, USA & Usual Care & 6 weeks & Depression (CES-D); Anxiety (STAI); Subjective Burden (ZBI) \\
\hline Wu, 2018, China & Usual Care & 12 weeks & Depression (HAMD); Anxiety (HAMA) \\
\hline
\end{tabular}

BSI: Brief Symptom Inventory; DASS: the 21-item Depression Anxiety Stress Scales; MHI: Mental Health Inventory; CHQ: General Health Questionaire; CESD: Center for Epidemiologic Studies Depression; STAI-State: State-Trait Anxiety Inventory-State; CRA: caregiver reaction assessment; BDI: Beck Depression Inventory; STAI: State-Trait Anxiety Inventory; HADS: Hospital Anxiety and Depression Scale; CQOLC: The Caregiver Quality of Life IndexCancer; ZBI: the Zarit Burden Inventory; HAMD: Hamilton Depression Scale; HAMA: Hamilton Anxiety Scale.

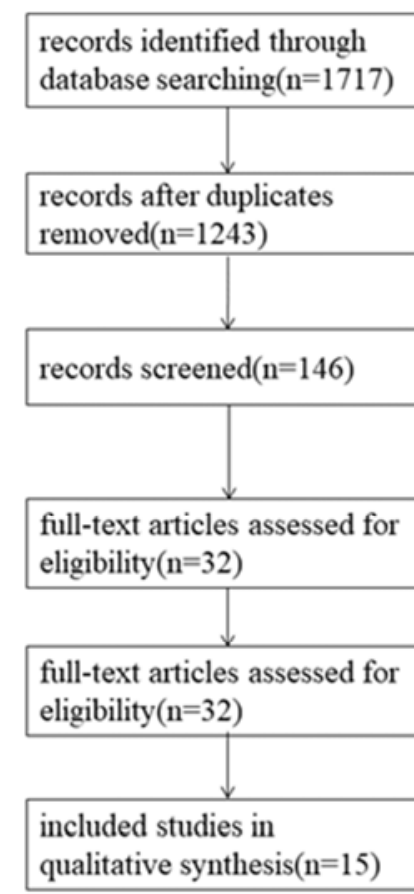

Figure 1. Flow diagram of trial selection. 


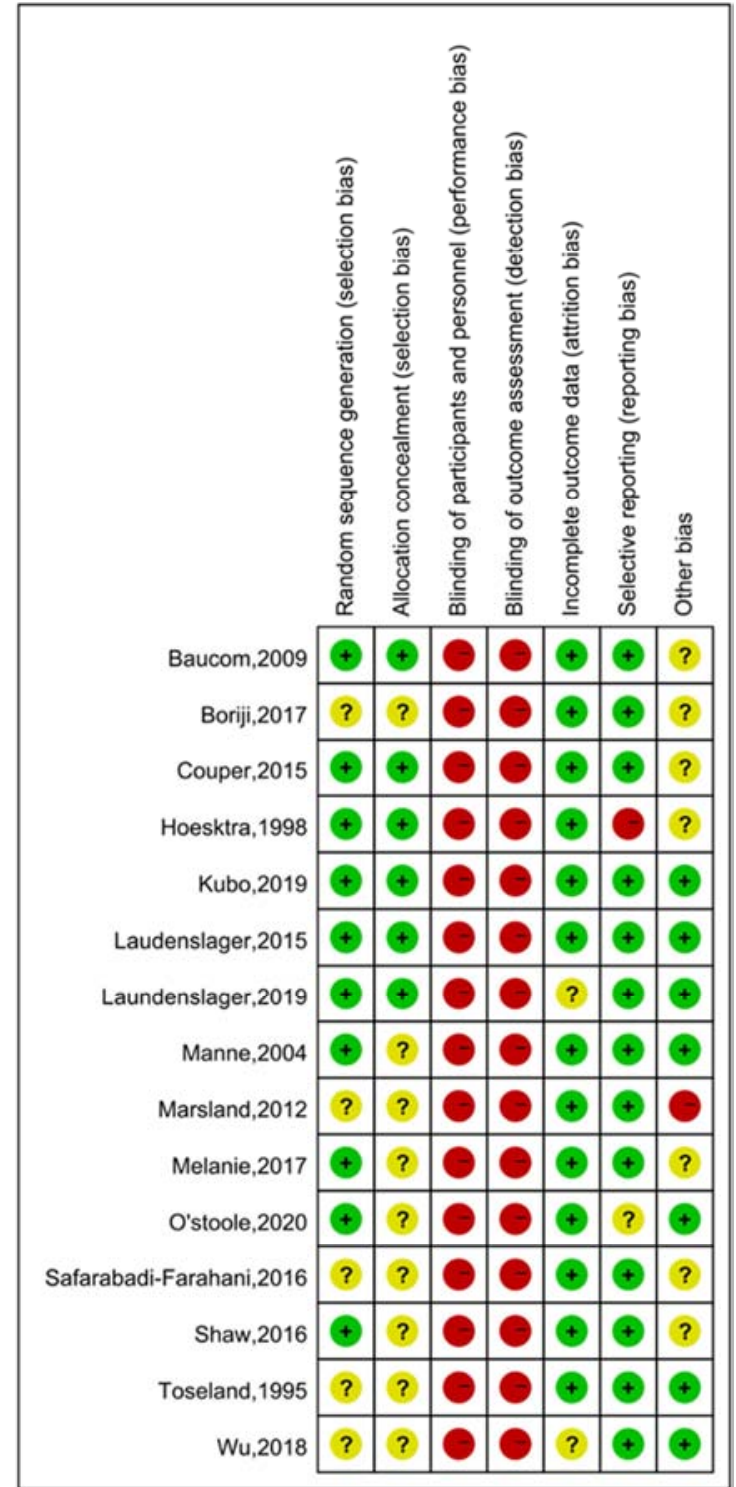

Figure 2. Risk of bias summary: review authors' judgements about each risk of bias item for each included study.

\subsection{Quality Assessment}

The included randomized controlled trials were all graded B for quality (Figure 2). Due to the particularity of psychosocial interventions, both the implementation of blinding of the participants and outcome assessment was impossible, so the performance bias and detection bias of all studies were assessed as high risks. Among the included fifteen RCTs, only 10 studies reported the methods for generating the random sequence $[5-7,12-15,17,18]$, and 6 studies describe the adequate method to conceal allocation $[6,7,12,13,15,18]$. Nine studies [5-7, 13, 15, 17-19, 21] reported shedding and withdrawing, of which seven studies $[5-7,13,15,17,21]$ applied the intent-to-treat analysis.

\subsection{Meta-analysis}

\subsubsection{General Psychological Distress}

Ten studies involving 690 participants assessed the outcome of general psychological distress (Figure 3.). Owing to the use of different measurement scales, the standardized mean difference (SMD) was selected. Significant heterogeneity was found between these studies $\left(\mathrm{I}^{2}=69\right.$, $\mathrm{P}=0.0005)$. The pooled results showed that the effect of the psychosocial intervention was not significant $(\mathrm{SMD}=-0.25$, $95 \% \mathrm{CI}-0.54$ to $0.05, \mathrm{P}=0.10$ ).

\subsubsection{Depression}

Among the included articles, six trials with 511 caregivers reported the depression outcome (Figure 4.). There was no heterogeneity among the 6 articles $\left(\mathrm{I}^{2}=40, \mathrm{P}=0.14\right)$. The difference between the intervention groups and the control groups was significant ( $\mathrm{SMD}=-0.47,95 \% \mathrm{CI}-0.71$ to $-0.24, \mathrm{P}<0.00001$ ), indicating that psychosocial interventions had a positive effect on reducing the depression level of cancer caregivers.

\subsubsection{Anxiety}

Six of the fifteen included studies measured indicators of anxiety, with 511 subjects overall (Figure 5). We found significant heterogeneity across the six articles $\left(\mathrm{I}^{2}=80\right.$, $\mathrm{P}<0.001$ ). The results, as shown in figure 5, suggesting that the psychosocial intervention was an effective strategy for eliminating the anxiety level of participants $(\mathrm{SMD}=-0.50$, $95 \%$ CI -0.91 to $-0.09, \mathrm{P}=0.02$ ).

\subsubsection{Subjective Burden}

Four articles assessed the subjective burden variable, including 396 participants (Figure 6). No heterogeneity was identified among the four trials $\left(\mathrm{I}^{2}=0, \mathrm{P}=0.54\right)$. It was found that there was no significant difference between psychosocial groups and usual care groups $(\mathrm{SMD}=-0.06,95 \% \mathrm{CI}-0.26$ to $0.14, \mathrm{P}=0.55)$.

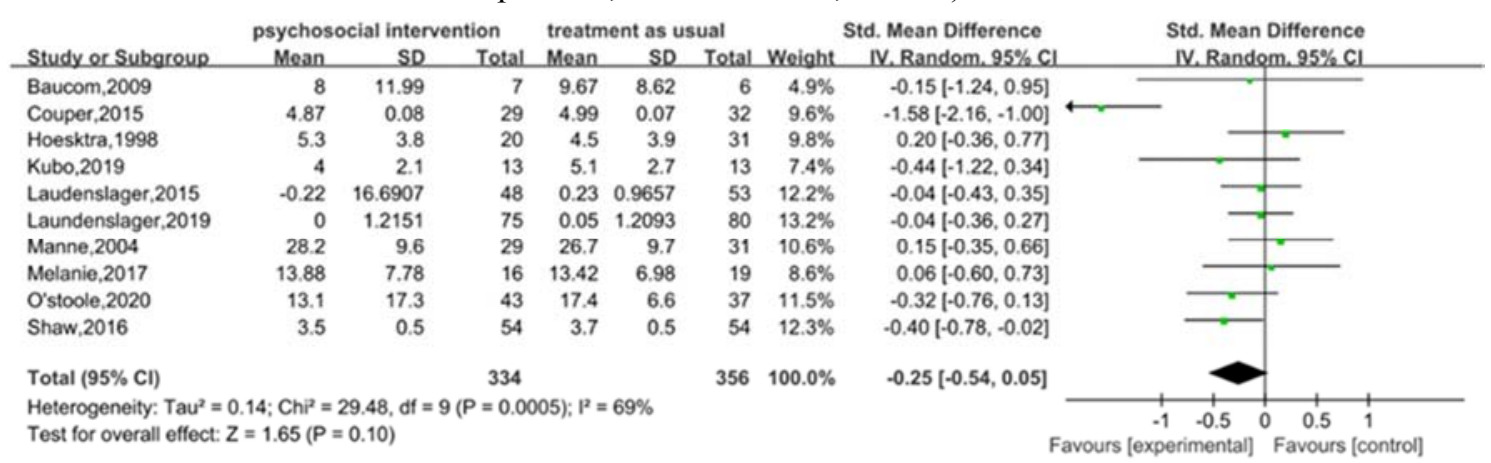

Figure 3. Forest plots effects of psychosocial intervention on psychological distress. 


\begin{tabular}{|c|c|c|c|c|c|c|c|c|c|}
\hline \multirow[b]{2}{*}{ Study or Subgreup } & \multicolumn{3}{|c|}{ psychosocial intervention } & \multicolumn{3}{|c|}{ treatment as usual } & & Std. Mean Difference & \multirow{2}{*}{$\begin{array}{l}\text { Std. Mean Difference } \\
\text { IV. Randem. } 95 \% \text { Cl }\end{array}$} \\
\hline & Mean & so & Total & Mean & so & Total & Weight & IV. Random. $95 \% \mathrm{Cl}$ & \\
\hline Boriji,2017 & 5.53 & 3.3 & 40 & 8.47 & 3.52 & 40 & $16.3 \%$ & $-0.85[-1.31,-0.39]$ & 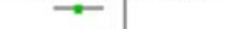 \\
\hline Laudenslager.2015 & 12.1 & 8.3068 & 48 & 16.3 & 8.543 & 53 & $19.4 \%$ & $-0.49[-0.89,-0.10]$ & - \\
\hline Laundenslager,2019 & 17.8 & 8.1742 & 75 & 20 & 7.7578 & 80 & $24.1 \%$ & $-0.27[-0.59,0.04]$ & $\rightarrow$ \\
\hline Marsland,2012 & 11.5 & 8.5 & 23 & 13 & 9.2 & 14 & $9.7 \%$ & $-0.17[-0.83,0.50]$ & \\
\hline Toseland,1995 & 16.3 & 6.2 & 40 & 17.7 & 6.7 & 38 & $16.9 \%$ & $-0.21[-0.66,0.23]$ & $\longrightarrow$ \\
\hline Wu,2018 & 4.77 & 2.79 & 30 & 8.83 & 5.9 & 30 & $13.5 \%$ & $-0.87[-1.40,-0.34]$ & 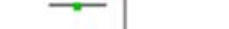 \\
\hline Total $(95 \% \mathrm{Cl})$ & & & 256 & & & 255 & $100.0 \%$ & $-0.47[-0.71,-0.24]$ & \\
\hline $\begin{array}{l}\text { Heterogeneity: } \mathrm{Tau}^{2}= \\
\text { Test for overall effect: }\end{array}$ & $\begin{array}{l}03 ; \mathrm{Chi}^{2}= \\
=3.91(\mathrm{P} .\end{array}$ & $\begin{array}{l}34, d f=5 \\
.0001)\end{array}$ & $=0.14)$ & $P^{2}=40 \%$ & & & & & $\begin{array}{lll}-1 & 0 & 1\end{array}$ \\
\hline
\end{tabular}

Figure 4. Forest plots effects of psychosocial intervention on depression.

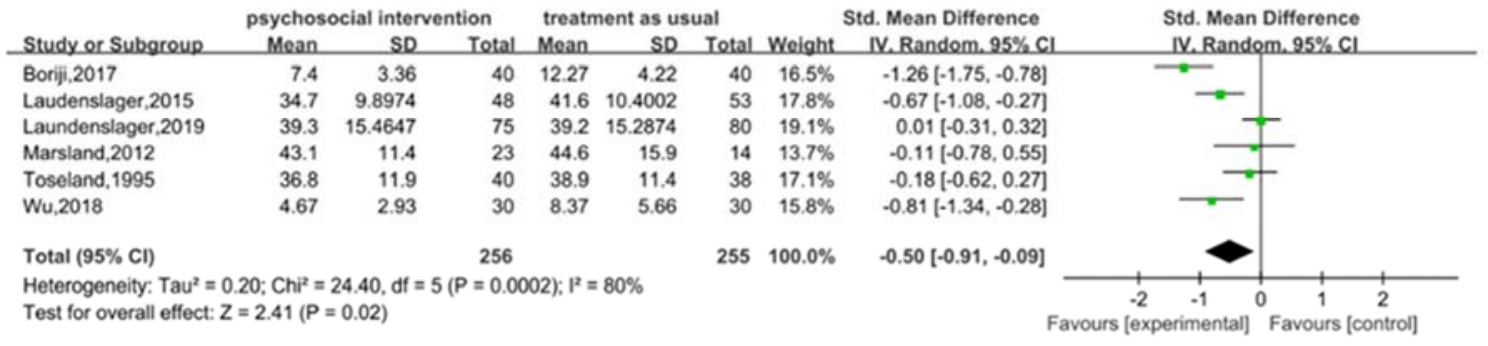

Figure 5. Forest plots effects of psychosocial intervention on anxiety.

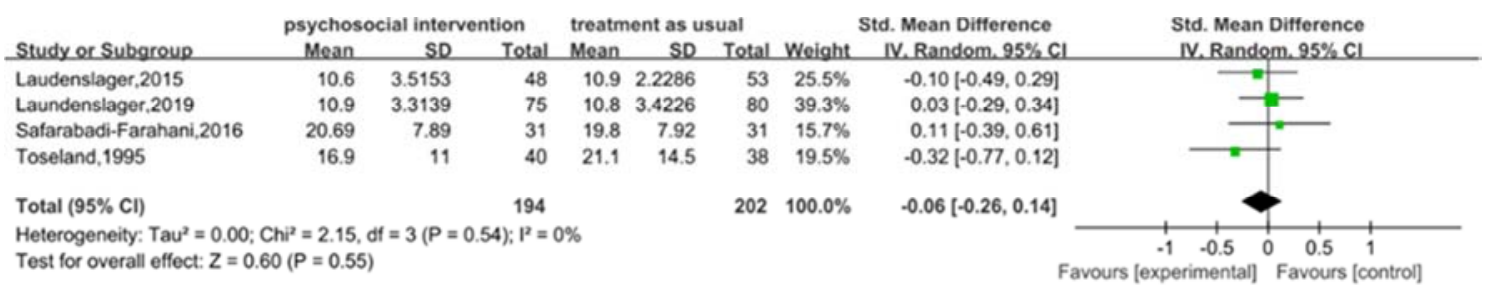

Figure 6. Forest plots effects of psychosocial intervention on subjective burden.

\section{Discussion}

With the increasing number of people diagnosed with cancer globally, more and more caregivers have to take the responsibility of providing care to cancer patients [22]. As revealed by meta-analysis, the levels of psychological distress in cancer caregivers are high [23, 24], which is detrimental both to the well-being of cancer caregivers and cancer patients. Researchers have conducted various types of psychosocial interventions to improve the mental health of cancer caregivers $[25,26]$, yet there is a dearth of related data.

Overall, this meta-analysis revealed that psychosocial interventions could reduce the level of depression and anxiety effectively among cancer caregivers, comparing with the usual care. The results are in accord with other meta-analysis studies indicating that psychosocial interventions for depression or anxiety are effective [27-29]. The purposes of psychosocial interventions are to help participants identify their dysfunctional behavior, teach them about cope strategies, thus maintain their well-being $[13,30]$. By proving psychoeducation interventions and psychological therapies, the level of depression, and anxiety of participants decreased.

However, we did not find any significant difference in the scores of general psychological distress and subjective burden between the two groups. A possible explanation for this might be the fact that the majority of participants were not severely distressed, thus the results showed no significant difference across the intervention groups and the control groups [14]. Another possible explanation is that distress declined over time for participants in both groups, and these group differences would be hard to detect by measurements [14].

Some limitations in this paper need to be mentioned. Firstly, some publications were missed because the literature search was limited to studies published in English or Chinese. Secondly, the bulk of trials was conducted in developed countries [5-7], lacking in evidence from developing countries. Thirdly, only six trials adequately described the process of randomization and allocation concealment, which might cause selection bias and assessment bias. Finally, clinical heterogeneity was inevitable due to the small sample size in several studies $[6,17,18,31]$, the disparity between the interventions $[6,20,30]$, and the variety among the outcome measures $[6,15,18]$.

\section{Conclusion}

The present study was designed to examine the effect of psychosocial interventions on reducing the level of psychological distress among cancer caregivers. Our study found that psychosocial interventions are effective for improving depression and anxiety in cancer caregivers. Future research should restrict the inclusion criteria concerning distress level, and implement interventions 
tailored to the cancer caregivers' needs. Besides, more studies with stronger methodological designs and larger samples are still needed.

\section{Conflict of Interest}

The authors declared no potential conflicts of interest.

\section{References}

[1] Cancer key facts. World Health Organization. 2018. Available at: https://www.who.int/news-room/fact-sheets/detail/cancer.

[2] Schulz R, Beach SR, Czaja SJ, Martire LM, Monin JK. Family caregiving for older adults. Annual review of psychology. 2020; 71: 635-659.

[3] Burnette D, Duci V, Dhembo E. Psychological distress, social support, and quality of life among cancer caregivers in Albania. Psycho-oncology. 2017; 26 (6): 779-786.

[4] Kim Y, Wellisch DK, Spillers RL, Crammer C. Psychological distress of female cancer caregivers: effects of type of cancer and caregivers' spirituality. Supportive Care in Cancer. 2007; 15 (12): 1367-1374.

[5] O'Toole MS, Mennin DS, Applebaum A, et al. A Randomized Controlled Trial of Emotion Regulation Therapy for Psychologically Distressed Caregivers of Cancer Patients. JNCI cancer spectrum. Feb 2020; 4 (1): pkz074.

[6] Kubo A, Kurtovich E, McGinnis M, et al. A Randomized Controlled Trial of mHealth Mindfulness Intervention for Cancer Patients and Informal Cancer Caregivers: A Feasibility Study Within an Integrated Health Care Delivery System. Integrative cancer therapies. Jan-Dec 2019; 18: 1534735419850634 .

[7] Laudenslager ML, Simoneau TL, Mikulich-Gilbertson SK, et al. A randomized control trial of stress management for caregivers of stem cell transplant patients: Effect on patient quality of life and caregiver distress. Psycho-oncology. 2019; 28 (8): 1614-1623.

[8] Badr H, Krebs P. A systematic review and meta-analysis of psychosocial interventions for couples coping with cancer. Psycho-Oncology. Aug 2013; 22 (8): 1688-1704.

[9] Tang WPY, Chan CWH, Leung DYP, Chan DNS. The effects of psychoeducational interventions on caregivers of children with cancer: A meta-analysis of randomized controlled trials. Journal of Child Health Care. Mar 2020; 24 (1): 123-142.

[10] Northouse LL, Katapodi MC, Song L, Zhang L, Mood DW. Interventions with family caregivers of cancer patients: metaanalysis of randomized trials. Ca A Cancer Journal for Clinicians. 2010; 60 (5): 317-339.

[11] Huedo-Medina TB, Sánchez-Meca J, Marín-Martínez F, Botella J. Assessing heterogeneity in meta-analysis: Q statistic or I2 index? Psychological Methods. 2006; 11 (2): 193.

[12] Hoekstra-Weebers JE, Heuvel F, Jaspers JP, Kamps WA, Klip EC. Brief report: an intervention program for parents of pediatric cancer patients: a randomized controlled trial. Journal of pediatric psychology. 1998-Jun 1998; 23 (3): $207-$ 214.
[13] Laudenslager ML, Simoneau TL, Kilbourn K, et al. A randomized control trial of a psychosocial intervention for caregivers of allogeneic hematopoietic stem cell transplant patients: effects on distress. Bone Marrow Transplantation. Aug 2015; 50 (8): 1110-1118.

[14] Manne S, Babb J, Pinover W, Horwitz E, Ebbert J. Psychoeducational group intervention for wives of men with prostate cancer. Psycho-oncology. 2004; 13 (1): 37-46.

[15] Couper J, Collins A, Bloch S, et al. Cognitive existential couple therapy (CECT) in men and partners facing localised prostate cancer: a randomised controlled trial. Bju International. 2015; 115: 35-45.

[16] WU Qianqian, LIN Xiaoji, CAI Limeng, et al. Study On Cognitive-Behavior Therapy for Improving the Mental Status among Family Members of Terminal Tumor Patients. Chinese Medical Ethics. 2018; 31 (06): 754-757.

[17] Schellekens MPJ, van den Hurk DGM, Prins JB, et al. Mindfulness-based stress reduction added to care as usual for lung cancer patients and/or their partners: A multicentre randomized controlled trial. Psychooncology. Dec 2017; 26 (12): 2118-2126.

[18] Baucom DH, Porter LS, Kirby JS, Gremore TM, Keefe FJ. A couple-based intervention for female breast cancer. PsychoOncology. 2010; 18 (3): 276-283.

[19] Safarabadi-Farahani A, Maarefvand M, Biglarian A, Khubchandani J. Effectiveness of a Brief Psychosocial Intervention on Quality of Life of Primary Caregivers of Iranian Children With Cancer: a Randomized Controlled Trial. Journal of pediatric nursing. 2016; 31 (4): e262-270.

[20] Toseland RW, Blanchard CG, McCallion P. A problem solving intervention for caregivers of cancer patients. Social science \& medicine (1982). 1995; 40 (4): 517-528.

[21] Shaw JM, Young JM, Butow PN, et al. Improving psychosocial outcomes for caregivers of people with poor prognosis gastrointestinal cancers: a randomized controlled trial (Family Connect). Supportive care in cancer. 2016; 24 (2 // *National Health and Medical Research Council* // 632645 (NHMRC) *National Health and Medical Research Council*): 585-595.

[22] Ferlay J, Soerjomataram I, Dikshit R, et al. Cancer incidence and mortality worldwide: sources, methods and major patterns in GLOBOCAN 2012. International journal of cancer. 2015; 136 (5): E359-E386.

[23] Hodges L, Humphris G, Macfarlane G. A meta-analytic investigation of the relationship between the psychological distress of cancer patients and their carers. Social science \& medicine. 2005; 60 (1): 1-12.

[24] Hagedoorn M, Sanderman R, Bolks HN, Tuinstra J, Coyne JC. Distress in couples coping with cancer: a meta-analysis and critical review of role and gender effects. Psychol Bull. 2008; 134 (1): 1.

[25] Waldron EA, Janke EA, Bechtel CF, Ramirez M, Cohen A. A systematic review of psychosocial interventions to improve cancer caregiver quality of life. Psycho-Oncology. 2013; 22 (6): 1200-1207.

[26] Northouse L, Williams A-1, Given B, McCorkle R. Psychosocial care for family caregivers of patients with cancer. Journal of Clinical Oncology. 2012; 30 (11): 12271234. 
[27] Minshall C, Pascoe M, Thompson D, et al. Psychosocial interventions for stroke survivors, carers and survivor-carer dyads: a systematic review and meta-analysis. Topics in stroke rehabilitation. 2019; 26 (7): 554-564.

[28] Akarsu N, Prince M, Lawrence V, Das-Munshi J. Depression in carers of people with dementia from a minority ethnic background: Systematic review and meta-analysis of randomised controlled trials of psychosocial interventions. International journal of geriatric psychiatry. 2019; 34 (6): 790-806.

[29] Jütten L, Mark R, Wicherts J, Sitskoorn M. The Effectiveness of Psychosocial and Behavioral Interventions for Informal Dementia Caregivers: Meta-Analyses and Meta-Regressions. Journal of Alzheimer's disease: JAD. 2018; 66 (1): 149-172.
[30] Borji M, Nourmohammadi H, Otaghi M, Salimi AH, Tarjoman A. Positive Effects of Cognitive Behavioral Therapy on Depression, Anxiety and Stress of Family Caregivers of Patients with Prostate Cancer: A Randomized Clinical Trial. Asian Pacific journal of cancer prevention: APJCP. Dec 28 2017; 18 (12): 3207-3212.

[31] Marsland AL, Long KA, Howe C, Thompson AL, Tersak J, Ewing LJ. A pilot trial of a stress management intervention for primary caregivers of children newly diagnosed with cancer: preliminary evidence that perceived social support moderates the psychosocial benefit of intervention. Journal of pediatric psychology. 2013; 38 (4): 449-461. 